\title{
Le récit journalistique au passé recomposé
}

\section{Préambule}

Comment démêler le vrai du faux? Comment séparer le tangible du vraisemblable? Comment distinguer un témoignage authentique d'une histoire rapportée? Comment valider la véracité d'un souvenir surgissant des brumes du passé ? Comment débusquer la certitude du réel dans l'éther de l'irréel ? Vastes questions aux mille réponses... Lesquelles taraudent autant qu'elles passionnent quantité de chercheurs. Et de journalistes.

Parfois qualifiés d'historiens de l'immédiat, ces derniers évoluent dans cette matière foisonnante de l'actualité, à savoir la somme des événements se déroulant dans l'instant le plus rapproché. Qu'il s'agisse d'une crise politique, d'une procédure judiciaire, d'un attentat terroriste, d'un conflit international, d'une découverte scientifique, d'un accord économique, d'une manifestation culturelle ou d'une compétition sportive, les professionnels de l'information sont tenus de rapporter ces événements dans leur immédiateté (parfois dans les conditions périlleuses du direct) pour informer au plus vite leurs différents publics.

Il arrive aussi que dans cet océan d'informations nouvelles, les journalistes soient amenés à privilégier une certaine durée, cette opportunité devenue de plus en plus rare aujourd'hui, en ces temps de disette économique. II s'agit le plus souvent d'une investigation nécessitant un investissement long et laborieux, destiné à révéler les ramifications de tel scandale financier, les dessous de telle affaire politico-judiciaire ou les origines de tel phénomène de société.

Enfin, ce journalisme du temps différé s'exerce également lors de faits d'actualité propices à un retour en arrière. On songera notamment aux multiples commémorations célébrant un événement majeur du passé. À titre d'exemple, en cette année du centenaire, la Première Guerre mondiale fut naturellement mise à l'honneur dans l'ensemble des médias. Comme on imagine l'année prochaine être fortement achalandée en reconstitutions diverses, destinées à faire revivre la Bataille de Waterloo. 
Pour ouvrir cette contribution destinée à évoquer le travail journalistique exercé au cœur des mémoires collectives, nous proposons au lecteur de revisiter la couverture rédactionnelle de certains épisodes finaux du dernier conflit mondial, lors des commémorations de 2004 et 2005 . Soit 60 années après la fin des hostilités. Responsable des opérations spéciales au journal Le Soir, nous avions coordonné plusieurs suppléments spéciaux et séries historiques qui nous avaient emmené dans le sillage de dizaines d'ainés ayant courageusement survécu à ces années de poudre et de plomb. Ils avaient à peine vingt ans, ce bel âge de l'insouciance qui annonce généralement un début de vie prometteur. Le leur fut impitoyablement balayé par la folie guerrière d'un régime totalitaire aux fondements démentiels.

Afin d'illustrer cette forme de journalisme aux accents rétroactifs, nous proposons d'examiner trois moments particuliers. En premier lieu, nous évoquerons le témoignage de quelques soldats impliqués dans l'espoir grandissant provoqué par le Débarquement en Normandie (6 juin 1944) et la libération progressive de l'Europe. Ce retour émouvant dans le passé se poursuivra à travers deux ressacs douloureux. On songe, bien évidemment, au sursaut d'Adolf Hitler lançant l'offensive von Rundstedt depuis l'est de la Belgique dans le but désespéré d'entamer une reconquête meurtrière qui allait incendier nos Ardennes (décembre 1944 et janvier 1945). Et surtout, on songe aux armées de la Libération qui allaient découvrir l'horreur des camps d'extermination nazis (Auschwitz est libéré le 27 janvier 1945 par l'Armée rouge) venant confirmer la barbarie inouïe engendrée par les acteurs hallucinés de la "Solution finale". Avant de conclure, nous évoquerons enfin un aspect particulier du métier d'informer à travers une enquête au dénouement surprenant.

Durant ces mois de couverture rédactionnelle, pour enrichir le propos, pour donner chair à la (vaste) documentation, nous avions privilégié le discours du vécu, celui de la parole et du témoignage. Ce choix nous était rapidement apparu comme une évidence, pour ne pas dire une priorité. L'âge avançant, la plupart de nos interlocuteurs ne ressentaient-ils pas l'extrême urgence de se raconter, de se livrer? Non pour se mettre en scène de manière ostentatoire et encore moins pour épater la galerie. Davantage encore que lors du $50^{e}$ anniversaire, ces témoins voulaient laisser une trace indiscutable de leurs jours de guerre, inscrite en lettres de peur et de sang. Et, avant tout, honorer la mémoire de leurs compagnons disparus, emmurés dans le silence strident des cimetières militaires et des fours crématoires. Mais surtout, chacun d'entre eux avait accepté d'entamer, avec autant d'émotion que de générosité, ce douloureux travail de mémoire qu'ils jugeaient impérieux de transmettre aux jeunes générations. Comme on rédige un testament bienveillant pour protéger l'avenir des siens. 


\section{La mémoire collective du Débarquement}

"Nous sommes partis d'ici! Nous avions creusé des trous individuels là-bas sous les pommiers. Les balles traçantes nous passaient au-dessus de la tête. C'était la première position que nous avions occupée. Nous avons eu des combats sérieux. Il y a eu six morts, dont le plus jeune d'entre nous qui est tombé le premier. " Abrité dans son imperméable beige soulevé par la brise marine, Jean Martial revit ces journées d'engagement intense, tout en embrassant du regard la campagne environnante de Salenelles, le premier village normand libéré par la brigade Piron en août 1944.

En ce mois de mai 2004, notre interlocuteur vient accomplir une reconnaissance pacifique sur les terres qu'il a libérées 60 ans plus tôt. Animé d'un sens de l'organisation remarquable, cet ancien mitrailleur bruxellois, retraité de la Sabena, prépare la venue de ses frères d'armes invités par les autorités normandes à commémorer le $60^{\mathrm{e}}$ anniversaire du Débarquement. Un périple que Jean Martial peaufine avec minutie puisqu'il faudra loger, nourrir et véhiculer une escouade de quatre autocars emplis de 200 vétérans, émus de retrouver la terre de leurs exploits. Avec un programme qui s'annonce serré. Chaque village, chaque municipalité tient à exprimer sa reconnaissance aux libérateurs qui n'ont malheureusement plus la forme éblouissante de leurs vingt ans. Et pourtant...

Embarqués le 6 août 1944, soit deux mois après le D-Day, ces 2.200 volontaires belges et luxembourgeois n'avaient jamais tenu un fusil en main. Solidement formés en Grande-Bretagne, ils avaient traversé la Manche à bord de quatre Liberty Ships pour débarquer, le 8 août, sur le sol normand encore secoué par de sérieux combats avec l'arrière-garde nazie. Quelque 500 véhicules et pièces d'artillerie avaient été déposés dans le port artificiel d'Arromanches pendant que les soldats se regroupaient un peu plus loin, autour du port de Courseulles-sur-mer. Placé sous commandement britannique, dirigé par le colonel Jean-Baptiste Piron, ce corps d'élite avait reçu comme ordre de mission de nettoyer la Côte fleurie entre Franceville-Merville et Honfleur, en passant par Houlgate, Cabourg, Deauville et Trouville. Le 4 septembre, la brigade Piron avait ensuite dû foncer vers Bruxelles pour défiler en tête du cortège des Alliés qui venaient de libérer la Capitale. Durant les mois suivants, ils allaient poursuivre leur difficile progression vers les Pays-Bas avant de contribuer à l'estocade finale du régime hitlérien aux portes de Berlin.

Aux côtés de Jean Martial, mémoire vivante de la brigade Piron, nous passerons cinq jours en Normandie pour découvrir cette route de la liberté ouverte par les soldats belges. Á force de rencontres avec les habitants, d'explications avec les édiles, d'anecdotes avec notre guide, nous prendrons pleinement la mesure du rôle capital joué par les libérateurs de la Côte fleurie. 
Genres majeurs du journalisme, le reportage et l'interview restent les instruments indispensables à la mise en forme vivante du récit. En croisant les sources, en multipliant les descriptions, en rappelant les circonstances, en décrivant les scènes et les lieux, le reporter s'attache à relater de manière concrète ce qu'il a vu et entendu. C'est la raison pour laquelle nous reviendrons, en août 2004, pour accompagner les vétérans dans leur excursion commémorative. Et passer énormément de temps avec ces personnes âgées dont la présence sur place met les sens en éveil pour mieux raviver l'acuité des souvenirs. On l'imagine assez, rien n'eût été pareil en se contentant de les rencontrer à leur domicile. Quelques scènes permettent d'illustrer ce travail de la mémoire hautement remuée par les circonstances. Mais dont il convient parfois de se méfier...

Après avoir traversé Francheville-Plage, le boulevard des Belges mène les vétérans au centre de Houlgate où trône une mairie en briques rouges. En face, un monument de grès rose salue les combattants des deux Guerres mondiales. Sur la gauche, on découvre une stèle gravée en lettres dorées : "À la mémoire des soldats de la première Brigade belge tombés à Houlgate le 21 août 1944 : Bekaert François, Betbeze Marcel, De Groote Hyppolyte, Gurhem Pierre, Jadon Lucien ». À l'issue de la cérémonie, on aperçoit une dame émue, un panier en osier coincé au creux du coude. Le 6 juin 1944, elle avait 19 ans : "Une de mes amies devait se marier ce jour-là! On a entendu crier : 'Ils arrivent!' Alors, on a annulé le mariage parce qu'on voulait vivre le Débarquement à la radio. Ici, ce sont les Belges de la Brigade Piron qui nous ont libérés. Je viens à chaque commémoration devant la stèle."

Autre lieu, autre souvenir... Nous voici à Deauville face au pont des Belges qui enjambe la Touques, orné d'une plaque rappelant la libération de la municipalité, le 22 août 1944. Larme à l'œil, submergé par la douleur, un ancien fantassin nous confie ce terrible épisode : "En GrandeBretagne, nous avions reçu un équipement extraordinaire mais nous étions déçus de ne pas avoir fait partie des premières troupes d'assaut. Notre tour est enfin venu deux mois plus tard. Le premier jour, j'ai eu très peur. Le deuxième, j'étais déjà habitué. À six kilomètres de Deauville, j’ai été envoyé en reconnaissance jusqu'à la Touques avec quatre autres soldats. Arrivés à la rivière, nous avons rencontré six résistants. Alors qu'ils nous renseignaient sur l'existence d'un fortin, un obus de 88 a explosé. Aussitôt après, j'ai vu les corps des résistants écrabouillés sur les murs. Et ce qui est terrible, c'est que cela ne nous touchait plus. II fallait avancer. Coûte que coûte». Un ange passe. À la vitesse d'une balle traçante. Et notre narrateur de poursuivre son récit, la voix chevrotante: «Nous nous sommes cachés dans une cave. Par le soupirail, on voyait un fusil mitrailleur sur le fortin. Alors qu'on pouvait attendre la troupe pour agir, mon copain a voulu y aller. II n'avait pas fait deux mètres qu'il avait déjà dix balles dans le corps ». 
En soirée, les anciens combattants belges sont invités à un banquet organisé en leur honneur au casino de Deauville. Une nouvelle occasion de prolonger les conversations, de recueillir des témoignages, de confronter les points de vue. Un léger doute va vite nous étreindre lorsque nous évoquons le tragique épisode du fortin que l'ancien fantassin venait de nous rapporter avec tant de sincérité, quelques heures plus tôt. "Cette histoire terrible s'est réellement passée mais celui qui vous l'a racontée ne figurait pas parmi les soldats qui l'ont vécue, nuancent deux vétérans compatissants. II l'a tellement entendue qu'il se l'est appropriée, sans doute de bonne foi. II n'a plus toute sa tête... ». Étrange fragilité du témoignage, mélangeant la vérité du réel au roman de l'irréel...

En prélude aux commémorations du 6 juin, un gigantesque travail de mémoire collective avait été également initié par les organisateurs du $60^{\mathrm{e}}$ anniversaire du Débarquement (le Mémorial de Caen) et la presse locale (le quotidien Ouest-France et France Bleu, l'antenne régionale de France Inter) avec le concours de la population normande. Durant des semaines, chaque ville, chaque village avait réuni ses habitants pour évoquer les années noires de l'Occupation et les combats de 1944 annonçant leur libération. Au total, 10.000 témoignages de civils et de vétérans avaient été recueillis, enregistrés et diffusés dans les médias partenaires.

En ce soir du 6 mai 2004, pile un mois avant le début des cérémonies officielles, nous arrivons au Mémorial de Caen pour assister à l'apothéose de cette entreprise mémorielle d'exception. Sous le regard réconfortant des portraits de Churchill, De Gaulle, Eisenhower et Montgomery, seize vétérans s'installent d'un pas lent sur l'estrade qui domine l'immense hall du Mémorial. Meilleurs alliés et pires ennemis se retrouvent côte à côte. Quatre militaires allemands côtoient douze représentants des armées libératrices (USA, Canada, GrandeBretagne, Pologne, France, Belgique...). Face à eux, quelque 1.600 invités, toutes générations confondues, les saluent dans un silence respectueux.

Lors de cette veillée inoubliable, l'heure de la réconciliation a déjà sonné entre les sans-grades de l'Histoire venus raconter leur été 1944. Un réveil de souvenirs émouvants, parfois drôles, souvent douloureux, qui rend pleinement sa force à la parole entendue, celle de ces survivants qui refusent le titre de héros pour mieux saluer la mémoire de leurs amis tombés dans leurs bras impuissants. Nièce de l'acteur Jean Gabin, Jacqueline Moncorgé se souvient de son retour des ÉtatsUnis où elle faisait ses études lorsque la guerre a éclaté : "Engagée comme agent de liaison entre les Alliés et l'administration française, j'ai rejoint l'Angleterre sur le Queen Elisabeth où 12.000 hommes étaient 
entassés. J'ai débarqué en Normandie, le 25 juin 1944. Depuis lors, j'ai conservé le souvenir d'un champ de bataille abominable. Des vaches mortes, pattes en l'air, jonchaient les champs. Un silence étouffant, une odeur de mort... Les maisons étaient en ruines. Tout avait été détruit. Le flux de réfugiés ne cessait pas. II fallait constamment créer des centres d'accueil. Il fallait rapprocher les familles, les aider à se retrouver, les soutenir moralement ".

En fin de soirée, nous nous approchons d'Anton Herr, ancien officier allemand de la Wehrmacht, revenu pour la première fois en Normandie, accompagné de son épouse, la fille d'un général allemand décédé. Ce commandant d'une division de panzers nous raconte "son " 6 juin 1944. Il figurait parmi les premiers blindés arrivés au combat. Comment douter de la force de ses souvenirs imprimés dans la tourmente du Débarquement? "Vers trois heures du matin, j'ai vite compris que le Jour J était arrivé. Entre deux vagues de bombardements aériens, mes douze blindés ont réussi à rejoindre Caen. Hormis les attaques sur les plages, il n'y avait pas encore eu de contact direct avec l'ennemi. Notre grande difficulté résidait dans le fait que le silence radio nous était imposé par les Américains qui s'était branchés sur notre fréquence. Alors que je hurlais depuis ma tourelle avec le lieutenant du char situé à dix mètres du mien, un obus a explosé et sa tête a été projetée sur moi ».

Et le vieux soldat de poursuivre son terrible récit venant corroborer les faits historiques, mais avec ce complément incomparable qu'ajoute le discours du vécu : «Oui, nous avons été très surpris! Hitler attendait le Débarquement dans le Pas-de-Calais. Beaucoup de nos généraux étaient partis ailleurs comme le maréchal Rommel qui était rentré en Allemagne pour l'anniversaire de sa femme. Finalement, ce qui m'a sauvé après plusieurs jours de combat, c'est que mon beaupère, le général Dollman qui commandait la 7e Armée, a osé enfreindre les ordres de Hitler en me disant de traverser les lignes ennemies pour regagner nos troupes à l'arrière. Fin juin, sur la route du Mans, j'ai aperçu mon beau-père qui était allongé dans sa voiture, tué d'une balle. Trente années plus tard, j'ai appris qu'il s'était suicidé. II n'avait pas supporté d'avoir désobéi à Hitler».

Submergé par l'émotion de cette soirée placée sous le signe de la réconciliation, Anton Herr nous confiera encore cette anecdote éloquente, favorisée par ce moment privilégié permettant l'émergence de son terrible passé : "Les contacts que j'ai eus aujourd'hui avec les autres vétérans ont été époustouflants! Ce matin, j'ai rencontré un Français qui avait 16 ans en 1944. II vivait dans le château que ma compagnie, la 21e Panzerdivision, occupait. Dans la nuit du 6 juin, des avions alliés avaient largué des tracts qui demandaient à la population 
civile de quitter les villes pour aller se réfugier dans les champs et éviter les bombardements intensifs. Ce jeune homme m'avait demandé ce qu'il devait faire. Je lui avais répondu qu'il serait préférable qu'il se réfugie dans les caves du château. Et aujourd'hui, il m'a remercié de lui avoir sauvé la vie. D'autres ont eu beaucoup moins de chance en se dispersant dans les campagnes...»

Étrange outil que cette mémoire qui peut aussi se souvenir de moments cocasses malgré l'enfer du Débarquement. En témoigne ce récit so british de Charles Hargrove (Grande-Bretagne), également interrogé au Mémorial de Caen : "Officier de liaison et interprète, j'avais reçu une jeep. Le problème, c'est que j'avais appris à conduire quatre jours avant le D-Day. J'avais une peur bleue de m'embrouiller avec les vitesses. Nous sommes restés coincés durant 48 heures dans notre péniche à cause de la tempête. On buvait du gin. Arrivés sur la Côte, on voyait la mer couverte d'embarcations. En attendant notre tour de débarquer, on repêchait des gars qui se noyaient tout en évitant les mines flottantes ». Et l'ancien officier d'en venir à ces détails qui font la différence : "Je suis sorti de ma péniche sans me mouiller les pieds, ce qui était devenu une obsession peu héroïque en ce jour de déluge. Sur la plage, le général de brigade m'a demandé de lui servir de chauffeur parce qu'il avait perdu sa jeep. Dans ses mémoires rédigés après la guerre, il avait écrit : 'En Normandie, il était plus dangereux d'être conduit par Charles Hargrove que de débarquer sous les obus'».

\section{La mémoire vivante de la Bataille des Ardennes}

Même à l'heure de la globalisation numérique, où circulent tant d'informations en cascade, le travail de terrain reste indispensable à la mission d'informer. Rares sont les reportages qui ne viennent s'enrichir d'une rencontre imprévue, d'un moment partagé, d'une opportunité provoquée. Après avoir publié de nombreux témoignages d'habitants ayant vécu la Bataille des Ardennes (hiver 1944-45), nous avions également couvert les cérémonies officielles de décembre 2004 à Bastogne, accompagné de nos confrères de la rédaction luxembourgeoise. Entre les multiples défilés, expositions, discours et cérémonies se déroulant dans des lieux divers, il fallait multiplier les angles de vue. Et tenter parfois de provoquer le hasard...

Le samedi 18 décembre, Bastogne vient de se réveiller sous la neige et les souvenirs. Grand-Rue, des anciens combattants battent le pavé avant de former un cortège qui se dirige vers le monument Patton où se dresse l'effigie du général américain sculptée dans le béton, un matériau à l'image du caractère carré de ce soldat d'élite. 
Parmi la délégation de 150 vétérans américains, une dame affligée aiguise notre regard. Renseignement pris, nous l'identifions avec certitude. Il s'agit d'Helen Patton, la petite-fille du lieutenant-général George Patton, disparu en 1945 à Diekirch, renversé par un camion dont les freins avaient lâché. En fleurissant la stèle de son grand-père, la jeune femme serre précieusement la canne de son aïeul, au pommeau gainé de cuir. Réflexe immédiat, nous tentons de l'approcher. "Je tenais à emporter un objet qui lui appartenait pour m'accompagner durant cette journée si importante ", nous souffle-t-elle dans un visage embué de larmes.

Peu après, les vétérans et les personnalités politiques se dirigent vers un second monument pour rendre hommage au général Mac Auliffe, cet autre héros qui avait défendu Bastogne encerclée par les nazis et lancé son fameux "Nuts" aux émissaires allemands venus lui poser un ultimatum. En remontant vers la place, Helen Patton insiste sur le caractère violent de cette bataille décisive pour l'issue de la Seconde Guerre mondiale : "Ici, en Ardenne, les combats ont été terribles, durs, sanglants. On l'occulte parfois. Tous ces soldats ont vécu des drames effroyables pour défendre le camp de la liberté ". La cérémonie débute. La discussion prend fin. Mais le hasard va en décider autrement.

Deux heures plus tard, nous devons interviewer Gérard Deprez, originaire de Noville, où sa famille a dramatiquement subi la bataille des Ardennes. Installée à la table du restaurant où l'ancien patron du PSC déjeune avec des élus locaux, Helen Patton nous salue. La discussion improvisée du matin peut alors se poursuivre pour en devenir plus intéressante encore. Symbole surprenant de la réconciliation entre ennemis d'hier, Helen Patton-Plusczyk nous apprend qu'elle vit dans la Sarre avec son mari allemand: "Mon mari vénère mon grand-père. Aujourd'hui, on commémore les soldats américains, mais il ne faut pas oublier tous les soldats allemands qui ont aussi perdu leur vie. Avec mon mari, nous nous battons quotidiennement pour que le nationalisme, qui peut conduire à de telles folies, soit maintenu à son niveau le plus bas. Nous avons créé une fondation artistique qui défend ces idées de respect, de liberté et de tolérance ".

Une autre coïncidence nous permettra de revenir sur un épisode fameux de la Bataille des Ardennes. En interrogeant certains vétérans belges venus à Bastogne, nous faisons la connaissance de Marcel D'Haene, un ancien engagé volontaire dans les Forces alliées. «Notre 5e bataillon a été créé le 9 octobre 1944 à Charleroi, explique le président de la fraternelle. Et le destin a voulu que nous nous soyons retrouvés en décembre dans la Bataille des Ardennes. " Au fil de la discussion, un détail nous interpelle. Marcel D'Haene nous annonce, 
à regret, que le prochain défilé du 21 juillet sera le dernier pour ces vétérans: "Ce sera la der des ders car les autorités estiment que nous n'avons plus la santé ». Et nous lui proposons de nous revoir, six mois plus tard, pour les accompagner dans leur ultime parade patriotique.

Comme convenu, le jour de la Fête nationale, Marcel D'Haene nous reçoit à l'heure de l'apéro dans son appartement d'Auderghem, au milieu de cinq camarades de combat. «Non merci, je ne bois rien. Je ne veux pas devoir soulager ma vessie pendant le défilé ", plaisante l'un d'entre eux. Au milieu de ces gamins de quatre-vingts printemps, la conversation fuse à la vitesse d'un obus de 75 perçant le blindage d'un panzer. Soixante années plus tard, ces fusiliers se souviennent aussi de la déconvenue infligée au sulfureux colonel SS Joachim Peiper sur les hauteurs de Stavelot. Alors que cet officier fanatique avait entamé une percée fulgurante dans les Ardennes qui devait le conduire sur les berges de la Meuse, sa division de blindés était à court d'essence et devait rapidement faire le plein.

Seul rescapé de cet exploit, Robert Lemaire prolonge son récit, avec une verve rarement rencontrée dans les livres d'histoire : "Mon peloton devait garder des stocks de jerrycans qui étaient mis par tas, le long de la route, sur une douzaine de kilomètres. L'offensive avait débuté le 16 décembre. Dès le 18, Peiper entrait déjà dans Stavelot. Lorsque le dernier Américain est passé devant nous, on entendait les chenilles des panzers à 200 mètres qui gravissaient la côte de la Haute Levée. Nous étions restés à dix, tous volontaires, pour garder le dépôt et éviter que Peiper ne puisse faire main basse sur l'essence. Pour aller vite, on a troué quelques jerrycans avec nos baïonnettes et versé l'essence sur les trois premiers tas tout en arrosant la route. On a foutu le feu avec une allumette et on s'est planqué ».

Impressionné par ce gigantesque incendie qui lui barrait le passage, Peiper sera contraint de faire demi-tour. Bloqué par les paras de la $82^{\mathrm{e}}$ Airborne, ses blindés tomberont en panne du côté de Stoumont. Réservoirs à sec, Peiper et ses hommes se dissiperont dans la nature. À cause d'une allumette craquée à bon escient...

\section{La mémoire partagée d'Auschwitt-Birkenau}

Pour donner davantage de sens à son propos, tout journaliste se doit de privilégier un angle qui servira de fil conducteur à son reportage. Les contraintes de temps et d'espace le poussent à éviter la dispersion des idées pour lui préférer une approche focalisée sur une poignée d'acteurs et de scènes, emblématiques de la thématique rapportée. 
En ces journées glaciales de la fin janvier 2005, l'évidence surgit au milieu des flocons tournoyant dans l'air givré de la Pologne qui s'apprête à commémorer la libération des camps d'extermination. L'Airbus de la Défense nationale belge se pose sur le tarmac verglacé de l'aéroport de Cracovie. A son bord des dizaines d'adolescents et quelques rares rescapés du camp d'Auschwitz (Oswiecim en polonais) s'apprêtent à découvrir l'indicible. Comment ne pas relater ces échanges intergénérationnels entre ces lycéens de Braine-le-Comte et les derniers survivants de la Shoah revenus sur les terres de leur martyre?

Ville industrielle aux couleurs ternes, Auschwitz affiche la déprime d'un bled de Haute-Silésie au sol pauvre et marécageux, qui oscille entre glace et moiteur au gré des saisons. Les nazis ont toujours eu le don de sélectionner leurs décors. Devant l'entrée d'Auschwitz I, les jeunes se regroupent avant de pénétrer sur le site de cette ancienne caserne polonaise transformée en usine de la mort. Premiers frissons. Recroquevillée dans son manteau sombre, Céline reconnaît la maxime coiffant la grille d'entrée : "Arbeit macht frei » ("Le travail rend libre »). Elle l'avait apprise durant les cours d'histoire destinés à préparer ce voyage initiatique. "Rien qu'à voir ces barbelés, je suis dégoûté », lance un garçon à ses amis.

Emmenés par Alberto Israël, ancien rescapé, les élèves se dirigent vers le Block 11. «Ici, l'enfer de Dante, c'était le paradis! Personne ne peut y passer sans pleurer, souffle le vieil homme. Le Block 11 dévorait les vies comme un ogre insatiable». Alberto Israël s'adresse à nouveau à son jeune public médusé: "Vous voyez ici, entre ce Block de torture et celui des femmes aux fenêtres occultées, il y avait ces murs. Les SS y faisaient entrer une vingtaine de prisonniers. À l'abri des regards, ils en appelaient un pour le tuer à bout portant avant d'appeler le suivant. Vous imaginez ce que le vingtième a dû subir avant d'être à son tour exécuté ? ". À voir leurs visages pétrifiés, il ne fait aucun doute qu'ils imaginent ces instants d'effroi. "Inimaginable! Quels salauds!», fulmine Karim.

Dans les Blocks 4 et 5 , la tragédie du camp sétale devant les yeux stupéfaits des jeunes visiteurs qui prennent soudainement conscience de l'insaisissable dimension de l'Holocauste. Dans un silence retenu, ils décodent ces innommables vitrines où sont amoncelés les derniers objets abandonnés par les centaines de milliers de victimes des chambres à gaz. Des lunettes enchevêtrées en pagaille. Des montagnes de chaussures fatiguées par les années d'errance. A côté, des valises indiquant les noms et dates de naissance de leurs propriétaires. Derrière l'énormité des chiffres, les élèves découvrent des bouts de vie hâtivement tracés à la craie. Plus loin, ils observent un amas de cheveux étendu comme un mausolée capillaire. Vendus 50 pfennigs le 
kilo, ils servaient à tisser des couvertures ou à gonfler des paillasses. Les lycéens comprennent que, pour les bourreaux d'Auschwitz, même la mort pouvait devenir source de profit.

La tête surmontée de son calot d'ancien prisonnier, Henri Kichka guide les adolescents devant un bâtiment allongé surmonté d'une cheminée : le four crématoire, ultime étape après le gazage dans ces fausses douches où les nazis déversaient les cristaux meurtriers du gaz Zyklon B. À l'intérieur, le spectacle devient indescriptible. "Ces chariots emmenaient deux ou trois cadavres à la fois dans le four, explique Henri Kichka. Des prisonniers juifs portaient les dépouilles avant d'être gazés à leur tour parce qu'ils en savaient trop ».

"C'est ici que j'ai perdu ma famille. Cet endroit, c'est mon cimetière! », lance Alberto Israël lorsque la délégation belge arrive au camp de Birkenau, sur les hauteurs d'Auschwitz. Un sol blanc. Des baraques noires. Alignées dans la neige à perte de vue. Du ciel, on dirait un damier. De la terre, une vaste nécropole, froide, glaciale, sans sépultures. Au centre une voie ferrée ne mène vers nulle part, stoppée net derrière un bâtiment allongé, percé d'un porche. Comme une fenêtre au bout de la nuit. Probablement la dernière vision aperçue par les déportés, jetés des wagons arrêtés devant cette rampe maléfique où trônait le Docteur Mengele, sélectionnant les détenus d'un simple coup d'œil. À gauche, la mort immédiate. À droite, le sursis. Mais, comme le précise Alberto Israël, "presque tous sont partis en fumée ».

Le surlendemain, le 27 janvier 2005, la communauté internationale se retrouve à Birkenau pour commémorer la libération du camp d'extermination par les troupes soviétiques. Un vent glacial gifle cet espace gigantesque où plus d'un million de victimes furent exterminées. En cette fin d'après-midi, de recueillement, il y a là quelque 2.000 rescapés, 50 vétérans russes, des centaines de délégations juives, des anciens des ghettos, des officiels, une foule d'anonymes. Tout autour, un millier de journalistes venus des quatre coins de la planète. Juché sur une tribune de presse où les chaînes de télévision ont installé leurs plateaux, un envoyé spécial de $T F 1$ s'apprête à prendre la parole pour une intervention en direct avec Paris. Engoncé dans un manteau de fourrure d'une griffe célèbre de l'avenue Montaigne, sa principale préoccupation consiste à enguirlander la maquilleuse qui n'a pas suffisamment laqué sa chevelure blonde soulevée par les flocons. À chacun ses urgences...

Pour la première fois en ce lieu symbolique, se retrouvent fondus en un seul hommage une quarantaine de chefs d'État dont les présidents polonais (Alexander Kwasniewski), américain (George W. Bush), russe (Vladimir Poutine), français (Jacques Chirac) et israélien (Moshe Katsav) aux côtés du chancelier allemand (Gerhard Schroeder). La 
Belgique, quant à elle, est représentée par le Roi Albert Il et le Premier ministre Guy Verhofstadt. Soudain, un sifflement transperce l'air glacial suivi du vacarme d'un train arrivant à destination. Lâcher de vapeur, crissement de freins, ouverture brutale des wagons. Frisson. Silence. Chacun se souvient, dans un dialogue émouvant avec les siens. Dans le public, des bras se serrent, des larmes coulent, des visages se figent.

Les discours politiques s'enchaînent. Impériale, Simone Veil s'avance vers la tribune: "J'ai le cœur serré par l'émotion, lance l'ancienne présidente du Parlement européen, également rescapée de Birkenau. Il y a 60 ans, la plupart d'entre nous ont été emmenés dans les marches de la mort, dans lesquelles ils mourront de faim, de froid et d'épuisement. Sur cette rampe, des hommes, des femmes, des enfants ont été sélectionnés par le Docteur Mengele. Que seraientils devenus? Des philosophes? Des artistes? De grands savants? Ou plus simplement d'habiles artisans ou des mères de famille? Je pleure. Je ne pourrai jamais oublier. Nous n'étions que des morceaux, comme ils disaient ".

Malgré les doigts engourdis, il faut noter ces souvenirs poignants, ces paroles de réconciliation pour les transmettre le plus fidèlement possible aux lecteurs. Et parfois, lire dans les lignes interstitielles de ces hommages, l'incongruité de certains messages. Alors qu'il compare la menace terroriste à la furie destructrice des nazis, l'inflexible Vladimir Poutine semble confondre le lieu et la manière pour justifier sa politique musclée en Tchétchénie. Imperturbable devant ce qu'il vient d'entendre, le président israélien Moshe Katsav revient sur la tragédie vécue par la diaspora juive : «Nuit et jour, avec une précision sans faille, les Allemands ont fait tourner l'industrie du génocide, une entreprise du meurtre pour notre peuple en Europe. Je redoute que l'eau des rivières européennes ne charrie encore le sang des victimes de la Shoah».

D'une voix cristalline, un artiste juif entonne un chant qui transperce les cœurs. Président d'honneur du Centre communautaire laïc juif (CCLJ), David Süsskind nous confie : "C'est la prière aux morts, à la mémoire... Il y a tellement d'émotion dans ce chant. Tous ces discours, ce n'est rien. Le plus important, c'est que nous soyons tous là. C'est magnifique de voir tous ces gens, toutes ces nationalités enfin réunies ici à Auschwitz-Birkenau dans un même élan. C'est la première fois!»

La soirée d'hommage prend fin en ce lieu de mémoire, éclairé de torchères, à jamais devenu patrimoine mondial de l'inhumanité. II reste à rédiger un article à la hâte avant le bouclage. Dans le bus qui nous ramène à Cracovie, nous attaquons les premières lignes. Mais le froid a eu raison des batteries de notre ordinateur portable. Arrivé au ser- 
vice de presse, nous parvenons à trouver une prise de courant dans un couloir. À $22 \mathrm{~h} 30$ au plus tard, les papiers doivent être envoyés à Bruxelles. Il nous reste une heure. Au moment de la transmission, l'ordinateur se plante à nouveau. Trois jours de reportage restent coincés dans le disque dur. Nous réussissons à les transférer sur une disquette pour l'installer dans un PC libre de la salle de presse. L'absurdité de la situation se poursuit. Le clavier de cet ordinateur russe se décline en alphabet cyrillique... Impossible de retrouver les commandes nécessaires à l'envoi. Nous donnons un coup de fil à la rédaction qui s'impatiente. A Bruxelles, un informaticien rallume son PC et va nous guider dans la procédure en nous dictant chaque geste pour transférer le texte, l'enregistrer et l'envoyer avec succès. À quelques secondes près, les souvenirs poignants d'Alberto Israël et de Henri Kichka auraient failli se perdre dans la nuit des camps.

\section{La mémoire retrouvée d'un soldat britannique}

Cette histoire étonnante débute par un courrier énigmatique reçu, en mai 2005, à la rédaction du Soir : "Cher Marc, en 1944, j'avais 21 ans. J'étais opérateur radar dans la Royal Air Force. Mon unité se trouvait dans la zone américaine des Ardennes. Lors de la contre-offensive allemande de décembre, nous avons été refoulés vers Mons où nous sommes restés durant quelques semaines. Pendant cette période, mes compagnons et moi avons été adoptés par des familles montoises. Par bonheur, j'ai été accueilli dans une adorable famille où il y avait une jeune fille dont je suis tombé amoureux. Je vous joins une photographie qu'elle m'avait dédicacée à l'époque. Je ne me souviens malheureusement plus de son nom. Mais j'aimerais tant savoir ce qu'elle est devenue. J'aimerais que vous puissiez la retrouver. Si elle est toujours vivante... Sincèrement vôtre et avec espoir. Eric Sutherland"

Postée du Surrey, en Grande-Bretagne, cette lettre aussi touchante que naïve ne peut qu'intriguer. Á commencer par la raison qui a guidé son auteur vers notre rédaction. II nous sera vite confirmé qu'il avait obtenu nos coordonnées via l'ambassade britannique de Bruxelles laquelle avait conservé nos écrits sur la Seconde Guerre mondiale. Pour quelles raisons, ensuite, l'épistolier ose-t-il confier à un obscur inconnu l'unique exemplaire d'une photo qu'il avait conservée comme une relique? Et enfin, comment retrouver, 60 années plus tard, une personne dont on ignore tout, jusqu'au nom, totalement illisible sur la dédicace de son portrait? Est-elle toujours en vie? Et dans l'affirmative, vit-elle toujours à Mons? Quant aux indices, ils sont aussi minces qu'une ration de guerre au siège de Bastogne. C'est dire si on part dans cette aventure avec un moral de vainqueur! Et c'est ainsi qu'allait débuter une enquête passionnante dans le passé ! 
Première étape indispensable, il nous faut vérifier la crédibilité de notre interlocuteur. Joint au téléphone, nous l'interrogeons longuement en lui demandant de remuer ses souvenirs, d'évoquer l'un ou l'autre détail utile à l'identification de la famille montoise. Tout au plus, se souviendra-t-il d'une maison située dans les faubourgs de la Cité du Doudou... Non sans ajouter cette précision qui va nous poser un solide problème déontologique : "Peut-être que nous avons eu un enfant... ". Si tel était le cas, nous sommes évidemment prêt à tout arrêter pour éviter de créer le moindre trouble au sein d'une famille. Certaines conséquences de la guerre se situent parfois au-delà des zones de conflit...

Après avoir rencontré la responsable du registre de la population de Mons, il nous est confirmé qu'une simple photo ne nous permettrait pas d'identifier la belle inconnue. II ne nous reste qu'à forcer la chance en tentant le tout pour le tout avec des anciens. Un après-midi d'été, nous sommes accueillis par le président des associations patriotiques de Mons. Nous lui expliquons la délicatesse de notre démarche et lui remettons des copies agrandies du cliché afin qu'il puisse les montrer à ses adhérents qu'il va rencontrer lors des cérémonies du 21 juillet. Peu après, le président nous appelle pour nous avouer son échec et s'apprête à nous renvoyer les photos. Nous l'invitons à les conserver et à persévérer. Entre-temps, nous tentons en vain de joindre Eric Sutherland pour faire le point. Sans succès. Nous apprendrons qu'il avait été hospitalisé pour remplacer son pacemaker. On croise les doigts. Pour lui. Pour elle. Peut-être...

Mi-août, le miracle se produit. Deux mamys viennent d'identifier l'inconnue avec certitude! Cette fois, on peut filer à l'hôtel de ville avec un nom. Et surtout, avec la certitude qu'aucun enfant ne soit né peu après la romance de l'hiver 1944-1945. On ressort avec une adresse. On appelle. On répond. Une voix d'homme, aimable, courtoise. Puis une autre. Plus douce, plus étonnée. Nous expliquons notre démarche. Rendez-vous est aussitôt pris.

Sourire timide, la dame nous reçoit en compagnie de son mari épousé en 1949 ("Je sais que ma femme a eu son petit succès", lance$\mathrm{t}$-il pour détendre l'atmosphère). Elle nous relate sa rencontre avec Eric Sutherland et Bill, son ami gallois : "Ma mère et moi revenions de la messe, un dimanche. Et nous avons aperçu deux soldats qui grelottaient dans le froid. Comme mon père avait rejoint la GrandeBretagne en 1940 et y avait été accueilli par une famille, ma mère était contente de pouvoir leur rendre ce service. Nous faisions leur linge, nous partagions nos repas ". Une romance débute avec le Britannique mais sera vite écourtée, en février 1945, avec le départ précipité des soldats en route vers Berlin : "J'avais un prétendant montois mais sa 
famille ne m'acceptait pas parce que nous n'étions pas issus du même milieu social. Je suis très touchée qu'Eric ait cherché à me retrouver après tant d'années. Dites-lui en tout cas que je n'ai pas épousé le prétendant de l'époque et que je suis très heureuse avec mon mari ».

Aussitôt dit, aussitôt fait. Depuis cette journée mémorable, Eric Sutherland a sa pression artérielle installée au beau fixe. Heureux de s'être retrouvés, ces deux-là communiquent par courriels. Et depuis lors, ils ont découvert une nouvelle ironie de leur étrange histoire : "L'un de mes deux fils a vécu en Angleterre où nous allions de temps à autre avec mon mari. Et ce qui est amusant, c'est que ses beaux-parents habitent à deux kilomètres de chez Eric Sutherland! C'est incroyable, nous aurions pu nous rencontrer par hasard! Toute cette histoire m'a rajeunie. Eric Sutherland viendra peut-être nous rendre visite. Quand je pense que vous m'avez retrouvée grâce à cette petite photo..." ".

\section{Conclusion}

Vécu avant tout comme une expérience sensible, le journalisme de terrain porte en lui les défauts originels de son éclosion. Parce qu'il sert avant tout à humaniser le propos, à étayer par le témoignage tout événement d'importance, à donner vie et sens à chaque histoire, il en contient toutes les fragilités. Chaque reporter le sait, l'admet et le réclame, l'art du récit mémoriel ressort d'une démarche à la fois volontaire et aléatoire. Volontaire dans les choix de s'intéresser à tel sujet de telle manière. Aléatoire dans la chaîne des opportunités qui s'offre à lui dans un espace donné à un moment donné.

Que valent les témoignages de quelques dizaines de personnes rencontrées pour illustrer un événement mondial ayant plongé des millions d'hommes et de femmes dans la tourmente des années de guerre? Peu de chose, il est vrai. Du moins en apparence. Car face à l'impossible exhaustivité des récits, face aux impératifs de publication, il ne reste souvent au journaliste que cette voie étroite des parcours particuliers pour tenter de frôler l'universalité des tragédies humaines. Et partager avec le public, la véracité d'une poignée de trajectoires significatives dont le tableau final ressemble davantage à une œuvre impressionniste, cet art sublime de la décomposition du réel, considéré comme dégénéré par le régime nazi. 